\title{
Adaptive quantization for spectrum exchange information in mobile cognitive radio networks
}

\author{
Arief Marwanto ${ }^{1}$, Sharifah Kamilah Syed Yusof ${ }^{2}$, Muhammad Haikal Satria ${ }^{3}$ \\ ${ }^{1}$ Department of Electrical Engineering, Universitas Islam Sultan Agung (UNISSULA), Indonesia \\ ${ }^{2}$ Department of Electrical Engineering, Faculty of Engineering, University Teknologi Malaysia, Malaysia \\ ${ }^{3}$ Department of Biomedical Engineering, University Teknologi Malaysia, Malaysia
}

\begin{tabular}{l} 
Article Info \\
\hline Article history: \\
Received Nov 6, 2018 \\
Revised Jan 16, 2020 \\
Accepted Jan 29, 2020 \\
\hline Keywords: \\
Adaptive quantization \\
Cognitive radio \\
Dynamic subcarrier mapping \\
Mu-law \\
Spectrum exchange information
\end{tabular}

Article Info

Received Nov 6, 2018

Revised Jan 16, 2020

Spectrum exchange information

\begin{abstract}
To reduce the detection failure of the exchanging signal power onto the OFDM subcarrier signal at uniform quantization, dynamic subcarrier mapping is applied. Moreover, to addressing low SNR's wall less than predetermine threshold, non-uniform quantization or adaptive quantization for the signal quantization size parameter is proposed. $\mu$-law is adopted for adaptive quantization subcarrier mapping which is deployed in mobility environment, such as Doppler Effect and Rayleigh Fading propagation. In this works, sensing node received signal power then sampled into a different polarity positive and negative in $\mu$-law quantization and divided into several segmentation levels. Each segmentation levels are divided into several sub-segment has representing one tone signal subcarrier number OFDM which has the number of quantization level and the width power. The results show that by using both methods, a significant difference is obtained around $8 \mathrm{~dB}$ compared to those not using the adaptive method.
\end{abstract}

Copyright (c) 2020 Institute of Advanced Engineering and Science. All rights reserved.

\section{Corresponding Author:}

Arief Marwanto,

Department of Electrical Engineering,

University Islam Sultan Agung (UNISSULA),

Kaligawe Street, KM 04, Semarang, Central Java, Indonesia.

Email: arief@unissula.ac.id

\section{INTRODUCTION}

Mobility makes CR node detects the power change at any time because every movement followed in the travel distance. According to [1-3] conducting a random movement activity in the CRN will do the un-conditional uniform quantization. Wherein the level of quantization and width is the same, just powers in detection are changed to the distance. As stated in [4] quantizing the local energy using numerous symbols regional sensing determination. The method has intended for low SNR signal detection problems. An interesting proposed method has shown in [5-12], the local decision statistics in the form of log-likelihood ratio (LLR) from individual detectors are quantized and sent to the fusion centre (FC). The statistical properties of the decision statistics in the presence of quantization are established. The quantized decision statistics are sent through a channel that may cause errors. The effect of channel errors is incorporated in the analysis through Bit Error Probability (BEP). The decision of hypothesis testing from individual sensing node detectors in the form of log-likelihood ratio are quantized and sent to the master node. The result of quantized decision will be sent via channel might causes error on reception at the master node. The errors on the channel can be analysis through bit error probability (BEP).

To optimize secondary transmit power allocation spectrum sharing, According to [13-18] has investigates the generalized Lloyds-type algorithm (GLA) [19-22] that utilizing quantized channel state information (CSI). A modified GLA is constructed for obtaining maximal power codebook, that proven to be generally merged and factually steady. The results describe that by using 3-4 bits of compensation, fully 
channel state information could achieve by modified GLA algorithm it makes ergodic capacity very close and uses only 4 bits of feedback. However, in [23] has shown that a good-conserve quantised which diminish mean square error of individual observation data's LLR at quantised outcome is gained by deploying Lloyd scheme. The quantised is designed by utilizing PDF of LLR of the sensing information. Numerical outcome shows that the GLA algorithm given equal sensing achievement against the initial LLR test by using a few quantization bits. To addressing bandwidth constraints, [23-25] has investigated optimizing quantization of LLR sensing data in cyclostationary signal detection using Lloyd-Max algorithm. The numerical results present that recommended design uses only less quantization bits which perform relatively the same sensing performance with coarse sensing observation, it's exceeded the arrangements with uniform quantised. Since uniform quantization adopted by $[2,23,26]$ an individual sensing doesn't impact to reduce bandwidth constraints and remains in overhead in control channel. Whereas, [23] has shown the reduction of quantization bits for saving the bandwidth. Those are proposed methods is not considering non-uniform quantization which leads to adaptive to the change of signal fluctuation received for individual $\mathrm{SN}$. Moreover, most of the proposed methods are not considering sampling signal and proceed in static mobility, this is become a challenge when sensing node is going to mobile.

\section{RESEARCH SYSTEM MODEL}

Explaining research chronological, including research design, research procedure (in the form of algorithms, Pseudocode or other), how to test and data acquisition [1, 3, 27]. The description of the course of research should be supported references, so the explanation can be accepted scientifically [4, 27]. In this works, a secondary mobile wireless networks (SN) represent a sensing node which sensed the PU spectral holes. At the $\mathrm{SN}$, the detected power level is exchanged (quantized) into one selected subcarrier number which represents the PU spectrum information on SN.

As shown in Figure 1, a centralized network is assumed utilized on the cognitive radio network topology. The nodes are moved in random speed which the velocity is $360 \mathrm{~km} / \mathrm{h}$ averaged. The nodes also move in random path; therefore, the phase angle is defined to be random. The distance is set to $0.1 \mathrm{~km}$ to 10 $\mathrm{km}$ which the SN nodes moving from the origin place towards the PU location as a destination. The PU is stationary. Since the mobility, path, and phase angle is random, the directional could be randomly moving toward the PU or farther from PU. Likewise, SN nodes travelled over the distances can be closer or farther from $\mathrm{MN}$ location, where the $\mathrm{MN}$ is stationary.

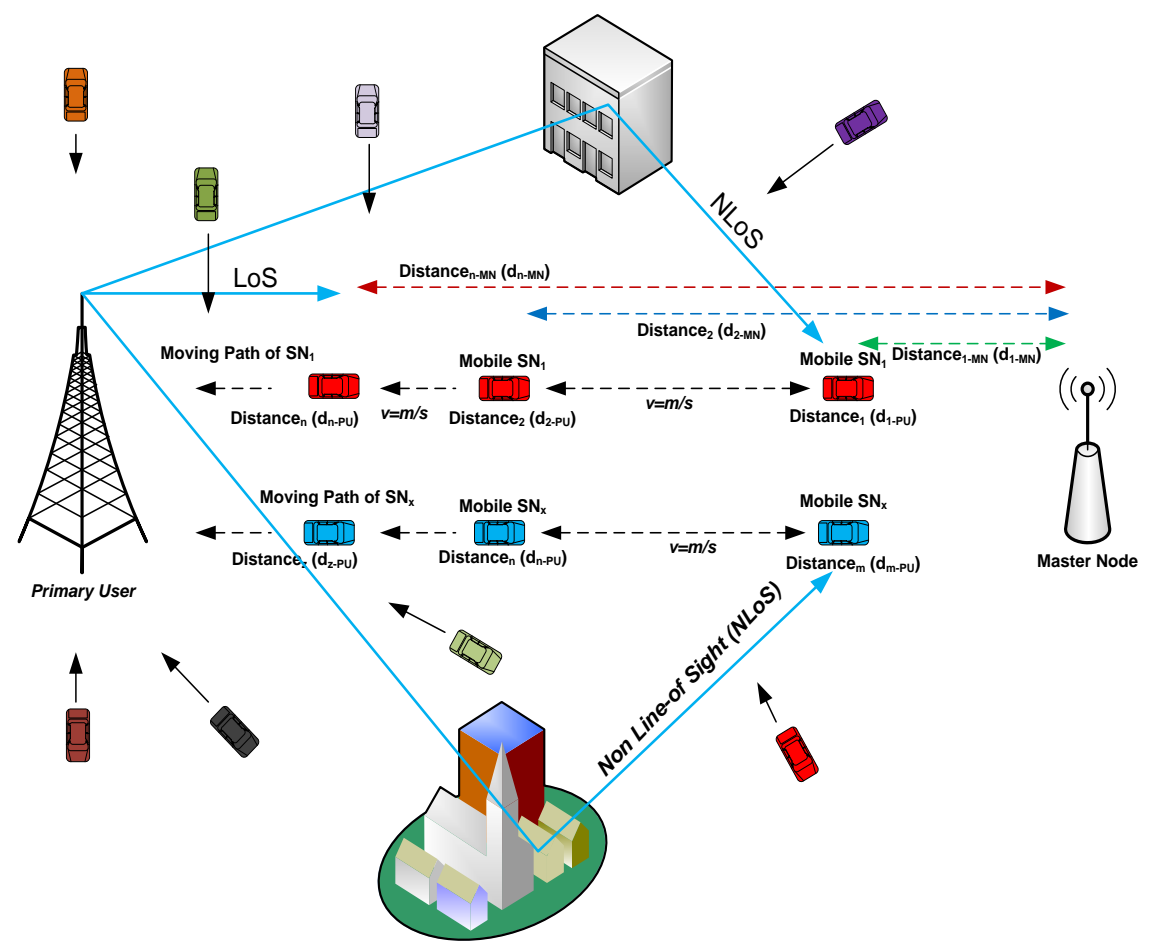

Figure 1. A scenario of the mobile spectrum exchange information [28] 


\section{3. $\quad \mu$-LAW ADAPTIVE QUANTIZATION ALGORITHM MODEL}

To perform quantization of the detection power level, each SN needs to sample the received signal of the radio wavelength energy into a discrete-time signal. Moreover, a sequence of sampling could deploy to sample the electromagnetic waves. The main function of sequence sample is to reduction of continuous signal which value is set at a point in time and/or space involves time periodically sensing within it sampler. An extraction of continuous signal is providing a quantization level and quantization interval in sensing process. Therefore, in one particular sensing process, the sampling is depending on the sample rate of the signal. Moreover, the sampling frequency or sampling rate, $f_{s}$, is the average number of samples obtained in one second (samples per second).

Previous method has shown un-conditional uniform quantization of the spectrum exchange information. Now, to addressing the limitation in previous works has shown the dynamic subcarrier mapping which able to manage intelligently subcarrier width accordance with the conditions of the detection power level. Therefore, the quantization results have a different width in accordance with the power detection for each node. Figure 2 shows the mechanisms of adaptive quantization for spectrum quantization information into OFDM subcarrier number. $\mu$-law is adopted for adaptive quantization subcarrier mapping based on the detected power level.

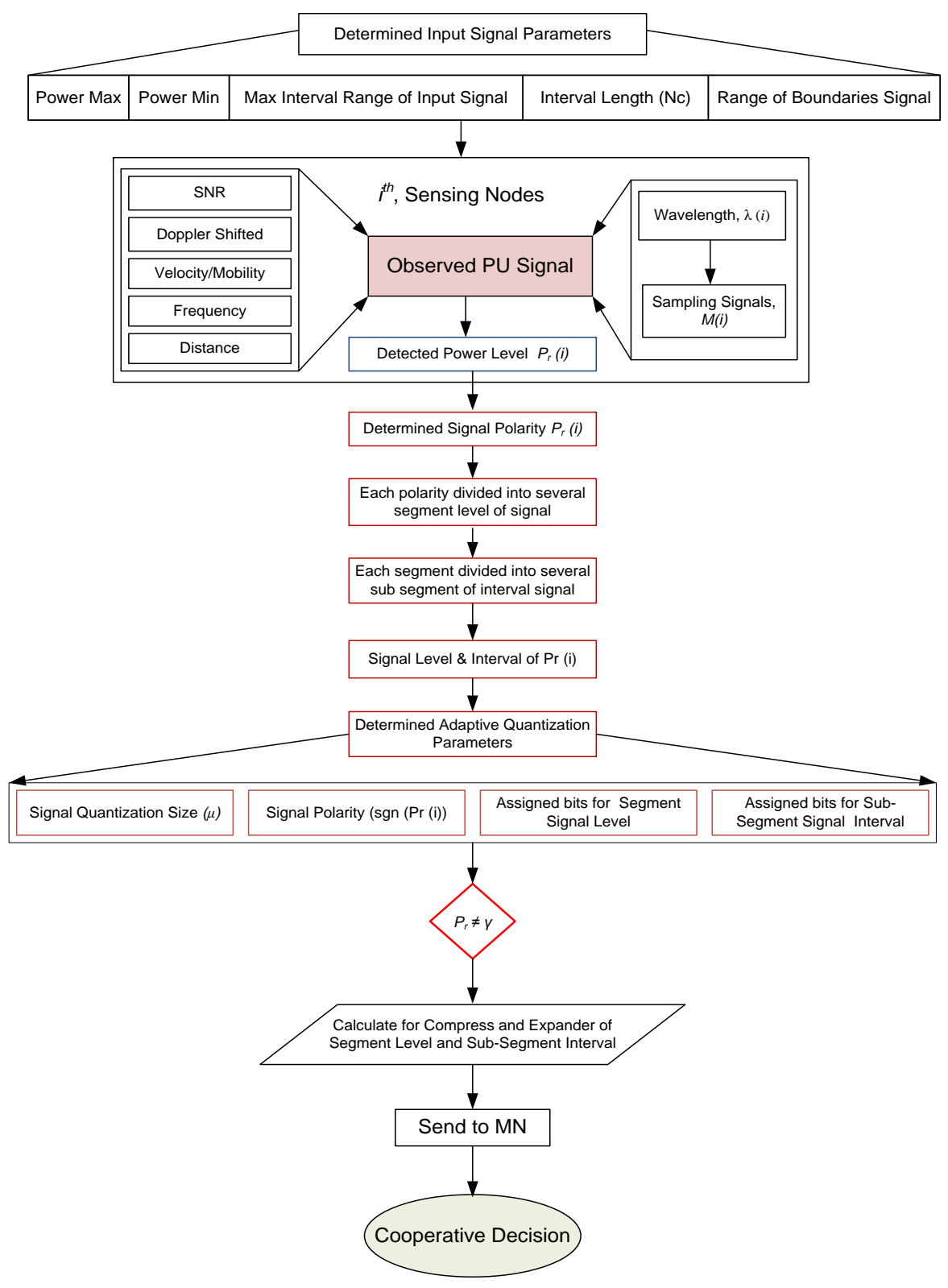

Figure 2. Adaptive quantization algorithm spectrum exchange information 
At the first stage, input signal parameters should be determined. These parameters including of the maximum minimum range of the detection power level $\left(P_{\max }\right.$ and $\left.P_{\min }\right)$, maximum number of the interval length $(I L)$, quantization intervals range $(I)$, and the range of the quantization intervals range of the input signal. In this work, the principle of quantization requires $-\infty=\mathrm{P}_{0} \leq \mathrm{P}_{1} \leq \ldots \leq \mathrm{P}_{M+1} \leq \mathrm{P}_{M}=\infty$ which determined ranges of detection power level in one particular sensing process. The range of detection power level is given by:

$$
P_{\min }=10^{-5} \text { and } P_{\max }=10^{-2}
$$

$P_{\min }$ is the pre-determined minimum of the detection power range of the input signal and $P_{\max }$ is the maximum of the detection power range of the input signal. The segmentation of each signal is required by determined the magnitude of the interval length (IL) in advance. The number of interval length is given by:

$$
I L=512
$$

In this term, the interval level is represented as subcarrier number OFDM. The interval length (IL) is also utilized to perform the quantization interval $(I)$ for segmentation of the received signal power, which given by:

$$
\begin{aligned}
& I=\frac{\left(P_{\min }+P_{\max }\right)}{I L} \\
& I=\frac{\left(10^{-5}+10^{-2}\right)}{512}=1.9551^{-5}
\end{aligned}
$$

Therefore, the interval (I) range that give for boundaries value among quantization resolution interval of the detection signal power. The range of the input signal is segmented by the quantization interval value which given by:

$$
\min _{P_{\text {Received }}}: I: \max _{P_{\text {Received }}}
$$

At the second stages, the sensing result is measured based on the average power of each SN node using which is sampled into non uniform variable coder. The received power is given by:

$$
\begin{gathered}
P_{R-\text { Mobile }}(i)=P_{T x} d B m+P_{T x \text { gain }}+S N_{\text {gain }}(i)-20 \log _{10}\left(\frac{\left.4 * \pi * d_{S N}(i) \Delta f_{R}\right)}{c}\right) \\
+r(t)(i)
\end{gathered}
$$

The maximum detection power level is sampled based on the quantization resolution interval signal value based on the interval length (IL) value. Every bit the results of the sampling signal is placed on the positive and negative polarity. Every polarity bits are divided into 512 segments or quantization signal level, which is given by:

$$
\begin{aligned}
& \text { segment }(\text { level })=S L=\left\lfloor\frac{\left(P_{\max }-P_{\min }\right)}{I}\right\rfloor \\
& S L=\left\lfloor\frac{\left(10^{-2}-10^{-5}\right)}{1.9551^{-5}}\right\rfloor \cong 512
\end{aligned}
$$

A segmentation of the input signal is defined as the value does not overlap in the set of real numbers. To make the segmentation as the criterion standard, the list of end point has different resolution in the vector. If $n$ is the length of the segmentation, then the index of vector $k^{\text {th }}$ entry is:

- $\quad 0$ if $\operatorname{sig}(k) \leq$ segmentation $(1)$

- $\quad \mathrm{m}$ if segmentation $(\mathrm{m})<\operatorname{sig}(k) \leq$ segmentation $(m+1)$

$-\quad \mathrm{n}$ if segmentation $(\mathrm{n})<\operatorname{sig}(k)$ 
The resolution intervals of the segmentation vector are one less than amount of the quantization interval. Commonly, an $M$-degree quantizer $(x)$ of an input value $x$ is denoted by a set of quantization degrees $l=\{l 1, \ldots, l M\}$ and quantization boundary $\mathrm{P}=\{\mathrm{P} 0, \ldots, \mathrm{P} M\}$. At the third stage, the segmentation levels are divided into 50196 sub-segments.

$$
\begin{aligned}
& s u b-\text { segment width }=s s w=\frac{\left(\max _{\text {power }} *\left(2 * \min _{\text {power }}\right)\right)}{I} \\
& s s w=\frac{\left(10^{-2} *\left(2 * 10^{-5}\right)\right)}{1.9551^{-5}}=0.0102
\end{aligned}
$$

Therefore, in one particular sensing cycle of the detection power level is comprised of:

$$
\begin{aligned}
& \text { Density }=D=\frac{\text { Number of Segmentations }}{0.0102} \\
& D=\frac{512}{0.0102}=50196 \text { segments }
\end{aligned}
$$

In such a way, sub-segments interval width could represent subcarrier mapping parameter and limited by segmentation level value as boundaries. Based on the sub-segments interval width calculation, the adaptive quantization for the detected power level is performed. The quantization of the detection signal into subcarrier number is given by:

$$
\text { quantiz }(\boldsymbol{K})=Q\left(P_{R}(i)\right) \text {. sign. } \Delta\left\lfloor\frac{\left|P_{R}(i)\right|}{\Delta}+\frac{1}{2}\right\rfloor
$$

The result is returned as third output parameter. Quantiz function generates quantization level index and the quantized output value. This function expressed as index of the input signal parameters to restore the quantization levels in real vector signal (sig) using the segmentation parameters. While, segmentation is a real vector signal that's inserted into specified order. Quantization is done to obtained the mapping signal $y=f(x)$ of areal signal into a discrete function valued. The basic principle is to make the resolutions steps that vary according to acceptable conditions in the signal. In this works, each sampling adopted difference resolution that maintains quantization is used to perform quantizing for individual SN users. The index quantization for segmentation level (IQL) is given by:

$$
\boldsymbol{I Q L}=Q\left(P_{R}(i)\right) . \operatorname{sign} . \Delta\left\lfloor\frac{\left|P_{R}(i)\right|}{\Delta}+\frac{1}{2}\right\rfloor\left(\left\lfloor 10^{\left(\frac{P_{R}}{20}\right)}\right\rfloor, \boldsymbol{S L}, \boldsymbol{S S W}\right)
$$

where $S L$ is segmentation level index, $S S W$ is sub-segmentation interval signal.

The next stage is determined adaptive quantization parameters. The parameters are quantization size mapping parameters for measurement value of the index signal level and interval width of received power, signal polarity of received power, assigned bit for segmentation level, and an assigned bit for sub-segmentation level. The aim's is to make the dynamic range (different width of segmentation level of the signals) of the input signal power.

The process of companding (compression and expanding) is used to overcome poor of SNR level or to compress the signal more than pre-determined threshold. The companding is aimed to distinguish the density of the quantization signal adaptively, if the detection SNR is poor, quantization levels at lower signal power must be denser than the signal power at a higher level. If signals detection is higher than threshold, then the quantization level at higher level should be denser than low level signal power. The quantization of the segmentation signal level and interval is adaptively changes by:

$$
\operatorname{compand}(\boldsymbol{C})=m\left(P_{R}(i)\right)=\operatorname{sign}\left(P_{R}(i)\right)\left(\frac{\ln \left(1+\mu\left|P_{R}(i)\right|\right)}{\ln (1+\mu)}\right)
$$

where, $0 \leq P_{R}(i) \leq 1$; $\operatorname{sign}\left(P_{R}(i)\right)$ is -1 if $P_{R}(i)$ negative and 1 otherwise, $\mu$ is 512 bits which represent as quantization measurement size/width parameter for each segmentation level and $P_{R}$ is detected power level each SN node. The companding signal value $(\boldsymbol{C S} \boldsymbol{V})$ reference signal is given by: 


$$
\boldsymbol{C S V}(i)=\operatorname{sign}\left(P_{R}(i)\right)\left(\frac{\ln \left(1+\mu\left|P_{R}(i)\right|\right)}{\ln (1+\mu)}\right)\left(\left\lfloor 10^{\left(\frac{P_{R}}{20}\right)}\right\rfloor, \mathbf{I}_{\mathbf{L}}, \mathbf{P}_{\max }\right)
$$

By finding non-zero element value in row and column within indexing value, the quantization into subcarrier number index given by:

$$
\begin{aligned}
& \text { subcarrier index }=\boldsymbol{S}=\sum_{n=1}^{N} n=\frac{(1+N c) N c}{2}>\operatorname{sign}\left(P_{R}(i)\right) \\
& \left(\frac{\ln \left(1+\mu\left|P_{R}(i)\right|\right)}{\ln (1+\mu)}\right)\left(\left\lfloor10^{\left.\left.\left(\frac{P_{R}}{20}\right)\right\rfloor, \mathbf{I}_{\mathbf{L}}, \mathbf{P}_{\max }\right)}\right.\right.
\end{aligned}
$$

where $N \boldsymbol{c}$ is the subcarrier index of the reference signal; $\boldsymbol{I} \boldsymbol{L}$ is interval length by given $512 ; \boldsymbol{P}_{\max }$ is maximum detection PU power, $10^{-2}$.

\section{MOBILE SPECTRUM EXCHANGE INFORMATION BASED COOPERATIVE SENSING}

All the components parameter has been modeled as stated in previous subchapter. In this part, every mobile SN's nodes will receives some distributed power from primary users (PU), which is given by:

$$
\begin{aligned}
P_{R-\text { Dopp }}(i)=P_{T x} d B m+P_{T x \text { gain }}+S N_{\text {gain }}(i) \\
-20 \log _{10}\left(\frac{\left.4 * \pi * d_{S N}(i) \Delta f_{R}\right)}{c}\right)+r(t)(i)
\end{aligned}
$$

where the $r(t)$ is Rayleigh fading based on summing sinusoids with Jakes model [11].

$$
r_{I}(t)=\frac{1}{\sqrt{N}} \sum_{m=1}^{N} \cos \left(2 \pi f_{d} \cos \alpha_{m} t+a_{m}\right)
$$

and

$$
r_{Q}(t)=\frac{1}{\sqrt{N}} \sum_{m=1}^{N} \sin \left(2 \pi f_{d} \cos \alpha_{m} t+b_{m}\right)
$$

and

$$
r(t)=r_{I}(t)+j r_{Q}(t)
$$

$f_{d}$ is the Doppler shift, $a_{m}=b_{m}$ is the amplitude of the signal and $N$ is multipath components with angle of arrival $\alpha_{m}$ of the nodes. The detected primary signal power then exchanged into subcarrier number of OFDM know as quantization power process. The conventional quantization mapping of the spectrum exchange information at $i^{t h} \mathrm{SN}$ given by [1].

$$
k_{\text {old }}(i)=\left\lfloor P_{R}(i) * \frac{N_{c}}{\alpha}\right\rfloor
$$

$k$ is assumed is the function of power quantization at the frequency carrier, $f_{c}$. If assuming that the $f_{c}=f_{R} ; f_{R}$ is the function of frequency in Doppler Effect which given by:

$$
f_{R}=f_{c}+\Delta f_{D}
$$

If detected power is a function of frequency then the $k$ is a function of frequency, however, a movement and impairment of the channel could rise during the sensing process, therefore, the received frequency of new subcarrier $f k_{1}^{\prime}$ is shifted during mobility in $\Delta V_{r}$ speed, which resulted into $\Delta f_{R}$ area. Therefore, $k^{\prime}$ is given by:

$$
k_{\text {Dopp }}^{\prime}=\frac{\left(f_{k}+\Delta f_{R}\right)-f_{c}}{f_{i}}=1 \pm\left[\frac{\Delta f_{R}}{f_{i}}\right]
$$


where $f_{i}$ is subcarrier width frequency in Hertz; However, due to signal is non-linear therefore $f_{i}$ is distinction in varies, hence:

$$
k_{\text {Dopp }}^{\prime}=\left[1 \pm \frac{\Delta f_{R}}{f_{i}}\right] * k_{\text {Old }}
$$

where $f_{R}$ the maximum received frequency Doppler shifted at the $n^{\text {th }}$ sensing nodes [29],

$$
\text { if } f_{i} \gg \Delta f_{R} \text { then } k^{\prime}=k
$$

and

$$
\Delta \mathrm{f}_{\mathrm{R}}(\mathrm{i})=\mathrm{f}_{\mathrm{R}}-\mathrm{f}_{\mathrm{c}}
$$

\section{RESULTS AND ANALYSIS}

In this term, mobile spectrum exchange (quantization) is evaluated with two different methods. The first method is spectrum exchange information in mobility environment based on [1, 30] proposed method. The second method is mobile spectrum exchange information utilizing Doppler Effect parameter. Those methods will investigate in term of adaptive quantization as utilized. The aims are to explore the performances of the cooperative decision probability at the gathering information node. In this work, the subcarrier mapping parameter in term is fixed, where the subcarrier mapping is given by $\alpha=2$. Therefore, the equation (22) should utilize to perform the quantization detection power level into subcarrier number. In addition, the method that utilized Doppler Effect is utilized in (25), where $\alpha$ is stationary. In term of adaptive quantization, the subcarrier parameter is based on the in (22) for conventional method; and in (25) for the method that utilized Doppler Effect. Except at the last sub-chapter, the adaptive quantization method is evaluated by using dynamic subcarrier mapping for comparative study between conventional methods that utilized dynamic subcarrier mapping.

\subsection{An analysis of spectrum exchange information using adaptive quantization}

Figure 3 shows the performance of detection probability in mobile spectrum exchange information. In this stage, two methods are proposed to be evaluated. In [30] method is assumed that Doppler Effect is zero (Old $K$ OHTA) and while the other method is based on the performance Ohta model using Doppler Effect (Doppler K). Both methods also are evaluated by using adaptive quantization (Mu Old K OHTA and Mu Doppler $K$ ). Therefore, four methods are analysis to perform cooperative detection in MN. The velocity of the nodes is sets for $360 \mathrm{~km} / \mathrm{h}$ which the directional path and moving is randomized.

From the graph it is clear that the evaluation analysis for the first method is marked by blue line is averaged at the point on 0.99 . However, at high level of subcarrier threshold value, the detection is decreased to 0.908 . While utilizing Doppler Effect methods which marked by red circle line are almost the same with the performance of the method that unutilized Doppler Effect. As can be seen also on the graph, the methods which are utilizing both the Doppler Effect and the adaptive quantization method have performed the same level of detection probability. It's shown by the green line and red circle line on the graph. However, uses both methods were slightly better performance other than unutilized adaptive quantization, whenever the detection of subcarrier threshold value greater than $8 \mathrm{~dB}$.

In comparison performance, can clearly be seen between [1] method which utilized or unutilized the adaptive quantization, the detection probability was slightly lower than method that utilized Doppler Effect and adaptive quantization. It is correspondent to the average of the selected subcarrier power threshold, the method that utilized the Doppler Effect and adaptive quantization has detected below the predetermined the detection threshold rules. Therefore, the probability can perform better than unutilized both methods. As illustrated by the graph, Figure 4 shows the probability of false alarm that evaluates the spectrum exchange information is presented. As can be seen from the graph, the conventional method (Old $K$ OHTA) has the same probability of false alarm performance compare to the method which is utilized Doppler Effect. However, at the high level subcarrier detection threshold value start from $8 \mathrm{~dB}$, the false alarm become rose to 0.092. On other hand, the method that utilized the Doppler Effect and adaptive quantization perform lowest other than conventional methods. The same thing is shown in the graph, when the subcarrier detection threshold level at position $8 \mathrm{~dB}$, it was increased false alarm, but smaller than conventional method, less than 0.08 . 


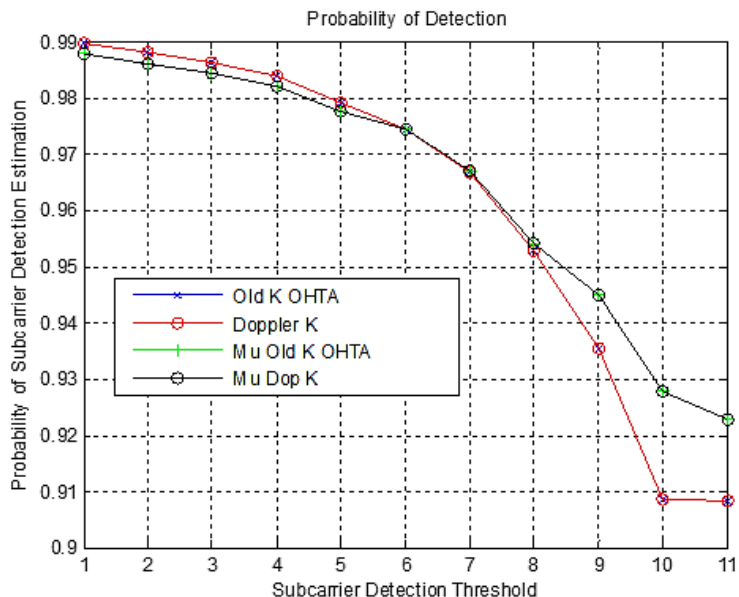

Figure 3. An analysis of detection probability from the mobile spectrum exchange information that utilize or unutilized adaptive quantization

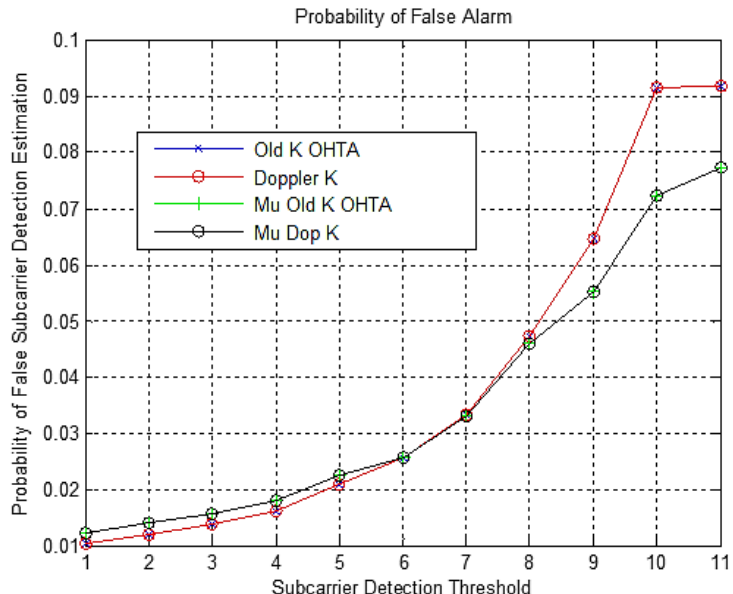

Figure 4. A comparative analysis study of false alarm probability for spectrum exchange information utilizing or unutilized adaptive quantization

\section{CONCLUSION}

It can be shown that the performance of adaptive quantization for spectrum exchange information in OFDM CR networks is presented. A comparative study also presented by compare the performance of two adaptive methods in spectrum exchange information. Both of adaptive methods based on the detection power level which is aim to obtain different width of quantization signal level. As previously mentioned, the adaptive quantization is able to perform well rather than conventional spectrum exchange information. Whereas, utilizing dynamic subcarrier mapping have the same performance with adaptive quantization. Moreover, the combining of dynamic subcarrier mapping and adaptive quantization have obtained the best performance in spectrum exchange information rather than if only dynamic subcarrier mapping or only adaptive quantization. For the future works, the combining of adaptive methods could be deployed in complexity model to investigated synchronization delay, time delay and subcarrier bit synchronization to combat the delay propagation effect within reporting channel. In a future, an experiment and testing are required to obtain the actual performance that can be applied in the real world.

\section{ACKNOWLEDGEMENTS}

This research was supported by Ministry of Higher Education of Indonesia (KemenRistekDikti) under World Class Professor (2018) Programme 2018, University Islam Sultan Agung (UNISSULA), University Teknologi Malaysia (UTM).

\section{REFERENCES}

[1] M. Ohta, T. Fujii, K. Muraoka, and M. Ariyoshi, "An OFDM based sensing information exchange for cooperative sensing in cognitive radio systems," Proc. SDR2008, 2008.

[2] M. Ohta, T. Fujii, K. Muraoka, and M. Ariyoshi, "A Novel Power Controlled Sensing Information Gathering for Cooperative Sensing on Shared Spectrum with Primary Spectrum," in Proceedings of the 6th International ICST Conference on Cognitive Radio Oriented Wireless Networks and Communications, pp. 111-115, 2011.

[3] H. Uchiyama et al., "Study on soft decision based cooperative sensing for cognitive radio networks," IEICE Trans. Commun., vol. 91, no. 1, pp. 95-101, 2008.

[4] L. Chen, J. Wang, S. Li, "Cooperative spectrum sensing with multi-bits local sensing decisions in cognitive radio context," in Wireless Communications and Networking Conference, 2008. WCNC 2008. IEEE, pp. 570-575, 2008.

[5] A. Marwanto, S. K. Syed Yusof, and M. H. Satria, "Orthogonal Frequency-Division Multiplexing-Based Cooperative Spectrum Sensing for Cognitive Radio Networks," TELKOMNIKA (Telecommunication Computer Electronics and Control), vol. 12, no. 1, pp. 143-152, Mar. 2014.

[6] H. A. Suraweera, P. J. Smith, and M. Shafi, "Capacity limits and performance analysis of cognitive radio with imperfect channel knowledge," IEEE Trans. Veh. Technol., vol. 59, no. 4, pp. 1811-1822, 2010.

[7] A. Sahai, N. Hoven, and R. Tandra, "Some fundamental limits on cognitive radio," in Allerton Conference on Communication, Control, and Computing, pp. 1662-1671, 2004.

[8] Y. Tani and T. Saba, "Quantization scheme for energy detector of soft decision cooperative spectrum sensing in cognitive radio," in 2010 IEEE Globecom Workshops, pp. 69-73, 2010. 
[9] K. Huang and R. Zhang, "Cooperative feedback for multiantenna cognitive radio networks," IEEE Trans. Signal Process., vol. 59, no. 2, pp. 747-758, 2010.

[10] Z. Tian, E. Blasch, W. Li, G. Chen, X. Li, "Performance evaluation of distributed compressed wideband sensing for cognitive radio networks," in 2008 11th International Conference on Information Fusion, pp. 1-8, 2008.

[11] A. Taherpour, M. Nasiri-Kenari, A. Jamshidi, "Efficient cooperative spectrum sensing in cognitive radio networks," in 2007 IEEE 18th International Symposium on Personal, Indoor and Mobile Radio Communications, pp. 1-6, 2007.

[12] S. Chaudhari and V. Koivunen, "Effect of quantization and channel errors on collaborative spectrum sensing," in 2009 Conference Record of the Forty-Third Asilomar Conference on Signals, Systems and Computers, pp. 528-533, 2009.

[13] X. Wang, Z. Li, P. Xu, Y. Xu, X. Gao, and H.-H. Chen, "Spectrum sharing in cognitive radio networks-An auction-based approach," IEEE Trans. Syst. Man, Cybern. Part B, vol. 40, no. 3, pp. 587-596, 2009.

[14] D. Niyato and E. Hossain, "A game-theoretic approach to competitive spectrum sharing in cognitive radio networks," in 2007 IEEE Wireless Communications and Networking Conference, pp. 16-20, 2007.

[15] X. Kang, Y.-C. Liang, H. K. Garg, and L. Zhang, "Sensing-based spectrum sharing in cognitive radio networks," IEEE Trans. Veh. Technol., vol. 58, no. 8, pp. 4649-4654, 2009.

[16] D. Niyato and E. Hossain, "Competitive pricing for spectrum sharing in cognitive radio networks: Dynamic game, inefficiency of nash equilibrium, and collusion," IEEE J. Sel. areas Commun., vol. 26, no. 1, pp. 192-202, 2008.

[17] D. Niyato and E. Hossain, "Competitive spectrum sharing in cognitive radio networks: a dynamic game approach," IEEE Trans. Wirel. Commun., vol. 7, no. 7, pp. 2651-2660, 2008.

[18] R. Zhang and Y.-C. Liang, "Exploiting multi-antennas for opportunistic spectrum sharing in cognitive radio networks," IEEE J. Sel. Top. Signal Process., vol. 2, no. 1, pp. 88-102, 2008.

[19] C. Senthamarai and N. Malmurugan, "Optimal Power Allocation for Outage Analysis Cognitive Full Duplex MultiChannel Relay Network Systems Using Multiple Objective Optimization Technique," J. Comput. Theor. Nanosci., vol. 15, no. 3, pp. 1004-1011, 2018.

[20] Y. Y. He, "Topics in resource optimization in wireless networks with limited feedback," PhD thesis, Engineering, Electrical and Electronic Engineering, The University of Melbourne, 2011.

[21] J. C. F. Li, W. Zhang, and J. Yuan, "Opportunistic spectrum sharing in cognitive radio networks based on primary limited feedback," IEEE Trans. Commun., vol. 59, no. 12, pp. 3272-3277, 2011.

[22] Y. He and S. Dey, "Power allocation in spectrum sharing cognitive radio networks with quantized channel information," IEEE Trans. Commun., vol. 59, no. 6, pp. 1644-1656, 2011.

[23] N. Nguyen-Thanh and I. Koo, "Log-likelihood ratio optimal quantizer for cooperative spectrum sensing in cognitive radio," IEEE Commun. Lett., vol. 15, no. 3, pp. 317-319, 2011.

[24] S. Bokharaiee, H. H. Nguyen, and E. Shwedyk, "Blind spectrum sensing for OFDM-based cognitive radio systems," IEEE Trans. Veh. Technol., vol. 60, no. 3, pp. 858-871, 2011.

[25] N. Nguyen-Thanh and I. Koo, "A cluster-based selective cooperative spectrum sensing scheme in cognitive radio," EURASIP J. Wirel. Commun. Netw., vol. 2013, no. 1, pp. 1-9, 2013.

[26] S. Chaudhari, J. Lundén, V. Koivunen, and H. V. Poor, "Cooperative sensing with imperfect reporting channels: Hard decisions or soft decisions?,” IEEE Trans. Signal Process., 2012.

[27] M. Ohta, T. Fujii, K. Muraoka, and M. Ariyoshi, "A Novel Power Controlled Sensing Information Gathering for Cooperative Sensing on Shared Spectrum with Primary Spectrum," 2011 6th International ICST Conference on Cognitive Radio Oriented Wireless Networks and Communications (CROWNCOM), pp. 111-115, 2011.

[28] A. Marwanto, S.K. Syed Yusof, and M.H. Satria, "Orthogonal Frequency-Division Multiplexing-Based Cooperative Spectrum Sensing for Cognitive Radio Networks," TELKOMNIKA (Telecommunication Computer Electronics and Control), vol. 12, no. 1, pp. 143, Mar. 2014.

[29] F. Xiong and M. Andro, "The Effect of Doppler Frequency Shift, Frequency Offset of the Local Oscillators, and Phase Noise on the Performance of Coherent OFDM Receivers," NASA Sci. Tech. Inf., vol. 2, no. 2, pp. 1-4, 2001.

[30] M. Ohta, T. Fujii, K. Muraoka, and M. Ariyoshi, "A Cooperative Sensing Area Expansion Using Orthogonal Frequency Based Information Collection Method," 2009 4th International Conference on Cognitive Radio Oriented Wireless Networks and Communications, 2009.

\section{BIOGRAPHIES OF AUTHORS}

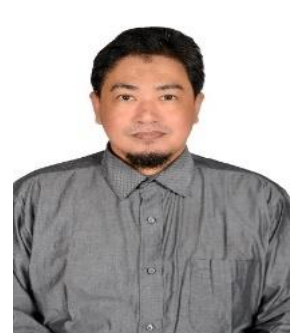

Arief Marwanto, received his Master and $\mathrm{PhD}$ in Electrical Engineering from Universiti Teknologi Malaysia (UTM). He became Lecturer at Department of Telecommunication Engineering, University Islam Sultan Agung (UNISSULA) Indonesia in 2001. Dr Arief has published over 50 articles in journals and conference papers and book chapter. His research interest includes the areas of wireless communications, in particular areas of Cognitive Radio, Internet of Things (IoT), Software Defined Radio (SDR), Software Defined Network (SDN), Biomedical Engineering and Application 
Sharifah K. Syed Yusof, received her BSc in Electrical Engineering from George Washington University, in 1988. She became Assistant Lecturer at the Department of Communication Engineering, Universiti Teknologi Malaysia (UTM) where she pursued her Master and PhD degrees at UTM respectively. Her research interest includes the areas of wireless communications, in particular areas of OFDM, Cognitive Radio, Wireless Sensor Network (WSN), Network Coding, Software Defined Radio (SDR), Software Defined Network (SDN) and Engineering Education. She has published over 150 articles in journals and conference papers. She is a co-editor of a book entitled Outcome-Based Science, Technology, Engineering, and Mathematics Education: Innovative Practices and several book chapters.

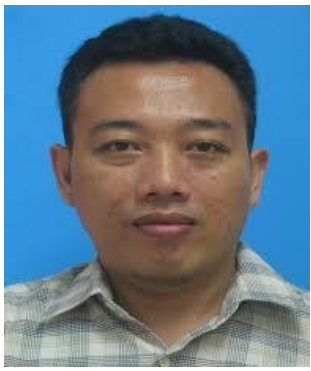

Muhammad Haikal Satria, received his Master in Computer Engineering from Universitaet Duisburg Essen Germany and $\mathrm{PhD}$ in in Electrical Engineering from Universiti Teknologi Malaysia (UTM). His research interest areas of Software Engineering, QoS Wireless Networks, Medical Informatics, Health Informatics, Healthcare Informatics, Telemedicine, Health Information Management, Medical Informatics Computing. He has published over 50 articles in journals and conference papers and book chapter. Currently he joined as Senior Lecturer at Department of Clinical Studies Faculty of Biomedical Engineering, Universiti Teknologi Malaysia (UTM). 